Applied Mathematical Sciences, Vol. 8, 2014, no. 57, 2833 - 2846

HIKARI Ltd, www.m-hikari.com

http://dx.doi.org/10.12988/ams.2014.417

\title{
An Attempt to Solve Decomposed Topology Optimization Problem Based Multicriteria Approach
}

\author{
K. Atani
}

LMI, FST Settat, BP : 577, Route de Casablanca, Settat, Morocco

A. Makrizi

LMI, FST Settat, BP : 577, Route de Casablanca, Settat, Morocco

\section{B. Radi}

LIMMII, FST Settat, BP : 577, Route de Casablanca, Settat, Morocco

Copyright (C) 2014 K. Atani, A. Makrizi and B. Radi. This is an open access article distributed under the Creative Commons Attribution License, which permits unrestricted use, distribution, and reproduction in any medium, provided the original work is properly cited.

\begin{abstract}
This paper deals with decomposed topology optimization problem (DTOP) written in [6] as a bilevel programming problem (BPP). The $\mathrm{BPP}$ are often known to be hard to solve in practice. Due to the effectiveness and efficiency of multicriteria techniques to solve industrial problems, several authors have attempted to establish a link between bilevel programming and multicriteria optimization [4]. For this reason, a finite element discretization procedure is described for DTOP, and the discrete problem is solved using multicriteria techniques.

In this context, we define an order relation such that the optimal solutions of BPP are the nondominated points with respect to this order relation. To achieve this outcome, we construct two convex cones, given the nondominated points with respect to the order relation induced by the cones, we prove that their preimage are nondominated with respect to the first order relation cited above, and are also solution to the BPP.
\end{abstract}

Mathematics Subject Classification: 74P05, 65M55, 91A65, 90C29 
Keywords: Topology optimization, domain decomposition method, bilevel programming problem, multicriteria optimization

\section{Introduction}

The bilevel programming problems BPP are a class of mathematical programming problems introduced and investigated by von Stackelberg [10], applied in the strategic game in economics, and occurs also in diverse applications, as transportation, engineering, optimal control, and others. BPP is set as hierarchical problems of two decision makers in which one, the so-called leader, has the first choice and the other one, the so-called follower reacts optimally on the leader's selection.

The conventional solution approach to the BPP is to transform the original two level problem into a single level one by replacing the lower optimization problem with the set of equations that define its KKT optimality conditions.

The multi-objective optimization or vector optimization framework allows optimizing many objectives simultaneously. In general, this problem has no single global solution. We use the notion of Pareto optimality to characterize solutions. The image of the efficient set (Pareto optimum) is called Pareto front and the contour of the Pareto surface indicates the nature of the tradeoff between the different objective functions [3].

In this work, we use the finite element approximation (FEA) to treat the decomposed topology optimization problem, written as a bilevel programming problem. Considering the complexity of bilevel programming problems BPP and the advantages of multicriteria techniques, we seek the solution of the BPP in the set of Pareto optimal points deducted through a non-dominance relationship defined on two convex cones. The efficient points or Pareto points of the multicriteria problem built from the BPP are the preimage of all nondominated points. A generalization of the relation between bilevel programming and multicriteria optimization was firstly developed by Fulop [5] for the linear case. Recently, A new relation based on the concept of nondominated points was proposed [4], for which, solving the BPP can be equivalent to solve a single optimization problem. In this context, we propose this approach for reformulating and solving our DTOP. Hence, the paper is organized as follows: in Sections 2 and 3 a description and finite element approximation of DTOP will be introduced and we specify the error estimation between the exact and approximate solutions. Section 4 is mainly concerned with the multicriteria formulation of approximated DTOP, we finish by a conclusion and perspectives. 


\section{Description of the DTOP}

Let $\Omega \subset \mathbb{R}^{2}$, be a smooth and bounded domain (of class $\mathcal{C}^{2}$ ) with a boundary $\partial \Omega$ which is divided in two disjoints parts $\Omega_{1}$ and $\Omega_{2}$ with the boundaries $\Gamma_{i}=\partial \Omega_{i} \cap \partial \Omega$, and $\Gamma_{0}=\partial \Omega_{1} \cap \partial \Omega_{2}$, is the interface between the two subdomains $\Omega_{i}$ (see figure 1). After decomposition of the domain, the DTOP takes the following form [6]:

$$
\begin{array}{cc}
\min _{\rho_{1}, \rho_{2}} \ell\left(u_{1}, u_{2}, g^{*}\right) & \\
\min _{g} \mathcal{J}_{\delta}\left(u_{1}, u_{2}, g\right) & \\
a_{\rho_{1}}\left(u_{1}, v_{1}\right)=\left(f, v_{1}\right)_{\Omega_{1}}+\left(g, v_{1}\right)_{\Gamma_{0}} & \forall v_{1} \in H_{\Gamma_{1}}^{1}\left(\Omega_{1}\right)^{2}, \\
a_{\rho_{2}}\left(u_{2}, v_{2}\right)=\left(f, v_{2}\right)_{\Omega_{2}}-\left(g, v_{2}\right)_{\Gamma_{0}} & \forall v_{2} \in H_{\Gamma_{2}}^{1}\left(\Omega_{2}\right)^{2},
\end{array}
$$

with the constraints on $\rho_{1}, \rho_{2}$ :

$$
\sum_{i=1}^{2} \int_{\Omega_{i}} \rho_{i}(x) d \Omega_{i} \leq V, \quad 0<\rho_{\text {min }} \leq \rho_{i}(x) \leq 1 \quad \forall x \in \Omega_{i}, \quad i=1,2 .
$$

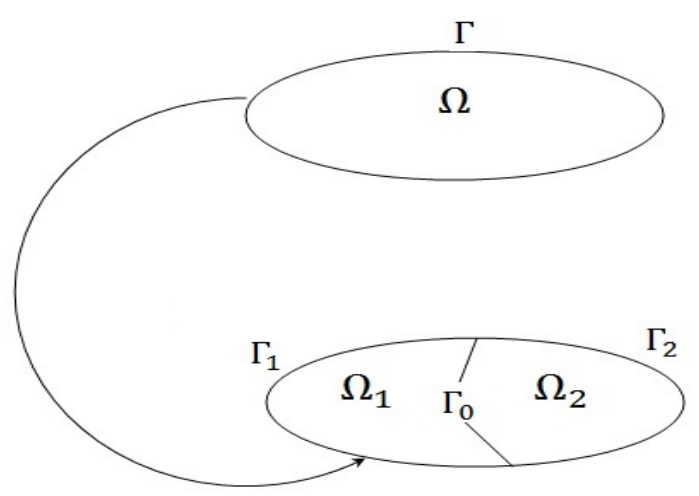

Figure 1: domain decomposition

the lower-level objective function is:

$$
\mathcal{J}_{\delta}\left(u_{1}, u_{2}, g\right)=\frac{1}{2} \int_{\Gamma_{0}}\left(u_{1}-u_{2}\right)^{2} d \Gamma_{0}+\frac{\delta}{2} \int_{\Gamma_{0}} g^{2} d \Gamma_{0}
$$

$" g^{*}=g^{o p t} "$ is the unique choice of $g$ for which $\left(g, u_{1}\right)_{\Gamma_{0}}=\left(g, u_{2}\right)_{\Gamma_{0}}, f$ is the force field, and $u_{i}(i=1,2)$ are the displacement field.

For $i=1,2, \rho_{i}$ are the density distribution corresponding to the subdomains $\Omega_{i}$, where a density of 1 would indicate the presence of material at a certain element, contrary to a density of 0 that means avoidance, a minimal density value, would indicate the presence of a void at the corresponding 
element. The upper-level objective function (compliance of the structure) is:

$$
\ell\left(u_{1}, u_{2}, g\right)=\int_{\Omega_{1}} f u_{1} d \Omega_{1}+\int_{\Gamma_{0}} g u_{1} d \Gamma_{0}+\int_{\Omega_{2}} f u_{2} d \Omega_{2}-\int_{\Gamma_{0}} g u_{2} d \Gamma_{0} .
$$

\section{Finite element approximation of the problem(1)}

\subsection{Discretization}

Given a triangulation $\mathcal{T}_{h}^{1}$ of the domain $\Omega_{1}$ composed of the triangles $K_{j}^{(1)}$, $j=1, \ldots, N_{e}$ with summits $\left(a_{k}\right)_{1 \leq k \leq N},\left(N_{e}\right.$ is the number of elements on each subdomain and $N$ denotes the number of nodes in $\left.\Omega_{i}, i=1,2\right)$. Similarly, $\mathcal{T}_{h}^{2}$ is a triangulation of the domain $\Omega_{2}$ composed of the triangles $K_{j}^{(2)}, j=1, \ldots, N_{e}$ with summits $\left(b_{k}\right)_{1 \leq k \leq N}$ and $\Gamma_{0}=\bigcup_{k=0}^{l-1}\left[a_{k}, a_{k+1}\right]$, where $\left(a_{k}\right)_{0 \leq k \leq l}$ are the nodes on the interface between $\Omega_{1}$ and $\Omega_{2}$.

Consider the discrete finite dimension subspaces: $\mathbb{P}_{k}$ is the set of polynomials of degree $\leq k$, and $C(\omega)$ is the space of continuous functions on a subset $\omega \subset \Omega$. For $i=1,2$ and $j=1, \ldots, N_{e}$,

$$
\begin{aligned}
\mathcal{W}_{i}^{h} & =\left\{v_{i}^{h} \in C\left(\bar{\Omega}_{i}\right) \mid v_{i \mid K_{j}^{(i)}}^{h} \in \mathbb{P}_{1}, \quad \forall K_{j}^{(i)} \in \mathcal{T}_{h}^{i}\right\}, \\
\mathcal{W}^{h} & =\left\{g^{h} \in C\left(\Gamma_{0}\right)^{N} \mid g_{i \mid\left[a_{k}, a_{k+1}\right]}^{h} \in \mathbb{P}_{1}, \quad \forall k=0, \ldots, l-1\right\}, \\
\mathcal{V}_{i}^{h} & =\left\{v_{i}^{h} \in \mathcal{W}_{i}^{h} \mid v_{i}^{h}=0 \text { on } \Gamma_{i}\right\}, \\
\mathcal{U}_{i}^{h} & =\left\{\rho_{i}^{h} \in L^{\infty}\left(\Omega_{i}\right) \mid \rho_{i \mid K_{j}^{h}}^{h} \in \mathbb{P}_{0}, \quad \forall K_{j}^{(i)} \in \mathcal{T}_{h}^{i}\right\}, \\
\mathcal{U}_{i}^{0, h} & =\left\{\rho_{i}^{h} \in \mathcal{U}_{i}^{h} \mid 0 \leq \rho_{i}^{h} \leq 1 \text { in } \Omega_{i}, \quad \int_{\Omega_{i}} \rho_{i}^{h}(x) d \Omega_{i} \leq \frac{V}{2}\right\},
\end{aligned}
$$

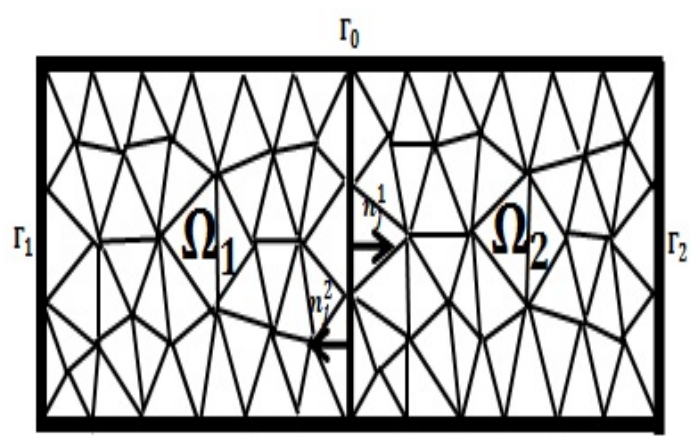

Figure 2: Meshing the subdomains $\Omega_{1}$ and $\Omega_{2}$ 


$$
g_{l}=\sum_{j=1}^{2} \sigma_{l j}\left(u_{1}\right) n_{j}^{1}=-\sum_{j=1}^{2} \sigma_{l j}\left(u_{2}\right) n_{j}^{2}
$$

with $g=\left(g_{l}\right)_{l=1,2}$ and $n_{j}^{i}$ denotes the normal vectors on $\Gamma_{0}$.

\subsection{Approximation}

Let $\left(\phi_{i}\right)_{1 \leq i \leq N}$ a basis of the subspace $\mathcal{V}_{1}^{h}$, with $\phi_{i}\left(a_{j}\right)=\delta_{i j}, \quad 1 \leq i, j \leq N$ and $\left(\psi_{i}\right)_{1 \leq i \leq N}$ a basis of the subspace $\mathcal{V}_{2}^{h}$, with $\psi_{i}\left(b_{j}\right)=\delta_{i j}, \quad 1 \leq i, j \leq N$. Let $u_{i}^{h} \in \mathcal{V}_{i}^{h}, i=1,2$ the approximated solution of problem (1), then:

$$
u_{1}^{h}(x)=\sum_{j=1}^{N} u_{1 j} \phi_{j}(x) \text { and } u_{2}^{h}(x)=\sum_{j=1}^{N} u_{2 j} \psi_{j}(x)
$$

where $u_{1 j}, u_{2 j}$ are the components of $u_{1}^{h}$ and $u_{2}^{h}$ in the basis $\left(\phi_{i}\right)_{1 \leq i \leq N}$ and $\left(\psi_{i}\right)_{1 \leq i \leq N}$.

The DTOP (1) can now be written as:

$$
\left\{\begin{array}{cl}
\min _{\rho_{1}^{h}, \rho_{2}^{h}} \ell\left(u_{1}^{h}, u_{2}^{h}, g^{* h}\right) & \\
\min _{g^{h}} \mathcal{J}_{\delta}\left(u_{1}^{h}, u_{2}^{h}, g^{h}\right) & \\
a_{\rho_{1}}\left(u_{1}^{h}, v_{1}^{h}\right)=\left(f, v_{1}^{h}\right)_{\Omega_{1}}+\left(g^{h}, v_{1}^{h}\right)_{\Gamma_{0}} & \forall v_{1}^{h} \in \mathcal{V}_{1}^{h} \\
a_{\rho_{2}}\left(u_{2}^{h}, v_{2}^{h}\right)=\left(f, v_{2}^{h}\right)_{\Omega_{2}}-\left(g^{h}, v_{2}^{h}\right)_{\Gamma_{0}} & \forall v_{2}^{h} \in \mathcal{V}_{2}^{h}
\end{array}\right.
$$

with the constraints on $\rho_{1}^{h}$ and $\rho_{2}^{h}$ :

$$
\sum_{i=1}^{2} \sum_{j=1}^{N_{e}} \int_{K_{j}^{(i)}} \rho_{i, j}^{h} d K_{j}^{(i)}-V \leq 0, \rho_{i, j}^{h} \text { is the density of material in } K_{j}^{(i)}, i=1,2
$$
and $0<\rho_{\min } \leq \rho_{i, j}^{h} \leq 1$.

By substituting $v_{1}^{h}$ for $\phi_{i}$ and $v_{2}^{h}$ for $\psi_{i}$, in (6), the lower level constraints become:

$$
\begin{aligned}
& a_{\rho_{1}}\left(u_{1}^{h}, \phi_{i}\right)=\left(f, \phi_{i}\right)_{\Omega_{1}}+\left(g^{h}, \phi_{i}\right)_{\Gamma_{0}} \quad \forall \phi_{i} \in \mathcal{V}_{1}^{h} \quad 1 \leq i \leq N \\
& a_{\rho_{2}}\left(u_{2}^{h}, \psi_{i}\right)=\left(f, \psi_{i}\right)_{\Omega_{2}}-\left(g^{h}, \psi_{i}\right)_{\Gamma_{0}} \quad \forall \psi_{i} \in \mathcal{V}_{2}^{h} \quad 1 \leq i \leq N \text {. }
\end{aligned}
$$

Hence, the approximations (5) yields

$$
\left\{\begin{array}{lll}
\sum_{j=1}^{N} a_{\rho_{1}}\left(\phi_{j}, \phi_{i}\right) u_{1 j}=\left(f, \phi_{i}\right)_{\Omega_{1}}+\left(g^{h}, \phi_{i}\right)_{\Gamma_{0}} \quad \forall \phi_{i} \in \mathcal{V}_{1}^{h} \quad 1 \leq i \leq N \\
\sum_{j=1}^{N} a_{\rho_{2}}\left(\psi_{j}, \psi_{i}\right) u_{2 j}=\left(f, \psi_{i}\right)_{\Omega_{2}}-\left(g^{h}, \psi_{i}\right)_{\Gamma_{0}} \quad \forall \psi_{i} \in \mathcal{V}_{2}^{h} \quad 1 \leq i \leq N
\end{array}\right.
$$


we pose the following expressions:

$$
\begin{array}{ll}
A_{1}^{h}=\left(a_{\rho_{1}}\left(\phi_{j}, \phi_{i}\right)\right)_{1 \leq i, j \leq N} & ; \quad A_{2}^{h}=\left(a_{\rho_{2}}\left(\psi_{j}, \psi_{i}\right)\right)_{1 \leq i, j \leq N}, \\
b_{1 i}^{h}=\left(f, \phi_{i}\right)_{\Omega_{1}}+\left(g^{h}, \phi_{i}\right)_{\Gamma_{0}} & ; b_{2 i}^{h}=\left(f, \psi_{i}\right)_{\Omega_{2}}-\left(g^{h}, \psi_{i}\right)_{\Gamma_{0}}, \\
b_{1}^{h}=\left(b_{1 i}^{h}\right)_{1 \leq i \leq N} & ; b_{2}^{h}=\left(b_{2 i}^{h}\right)_{1 \leq i \leq N} \\
u_{1}^{h}=\left(u_{11}, \ldots, u_{1 N}\right)^{t} & ; u_{2}^{h}=\left(u_{21}, \ldots, u_{2 N}\right)^{t},
\end{array}
$$

with these notations, the BPP (6) can be expressed as:

$$
\left(P_{h}\right)\left\{\begin{array}{c}
\min _{\rho_{1}^{h}, \rho_{2}^{h}} \ell\left(u_{1}^{h}, u_{2}^{h}, g^{* h}\right) \\
\min _{g^{h}} \mathcal{J}_{\delta}\left(u_{1}^{h}, u_{2}^{h}, g^{h}\right) \\
A_{1}^{h} \cdot u_{1}^{h}=b_{1}^{h} \\
A_{2}^{h} \cdot u_{2}^{h}=b_{2}^{h}
\end{array}\right.
$$

with the constraints on $\rho_{1}^{h}$ and $\rho_{2}^{h}$ :

$$
\sum_{i=1}^{2} \sum_{j=1}^{N_{e}} \rho_{i, j}^{h} \mu\left(K_{j}^{(i)}\right)-V \leq 0 \quad \text { with } 0<\rho_{\min } \leq \rho_{i, j}^{h} \leq 1
$$

where $\mu\left(K_{j}^{(i)}\right)$ denotes the measure of the element $K_{j}^{(i)}$.

The upper-level objective function (compliance of the structure) can be written as:

$$
\ell\left(u_{1}^{h}, u_{2}^{h}, g^{h}\right)=\int_{\Omega_{1}} f u_{1}^{h} d \Omega_{1}+\int_{\Gamma_{0}} g^{h} u_{1}^{h} d \Gamma_{0}+\int_{\Omega_{2}} f u_{2}^{h} d \Omega_{2}-\int_{\Gamma_{0}} g^{h} u_{2}^{h} d \Gamma_{0}
$$

replace $u_{i}^{h}$ by the expression (5), and put: $\varphi_{f j}=\int_{\Omega_{1}} f \phi_{j} d \Omega_{1} ; \varphi_{g j}=\int_{\Gamma_{0}} g^{h} \phi_{j} d \Gamma_{0}$ $; \psi_{f j}=\int_{\Omega_{2}} f \psi_{j} d \Omega_{2}$ and $\psi_{g j}=\int_{\Gamma_{0}} g^{h} \psi_{j} d \Gamma_{0}$, then,

$$
\ell\left(u_{1}^{h}, u_{2}^{h}, g^{h}\right)=\sum_{j=1}^{N} u_{1 j} \varphi_{f j}+\sum_{j=1}^{\ell+1} u_{1 j} \varphi_{g j}+\sum_{j=1}^{N} u_{2 j} \psi_{f j}-\sum_{j=1}^{\ell+1} u_{2 j} \psi_{g j}
$$

the lower-level objective function becomes:

$$
\mathcal{J}_{\delta}\left(u_{1}^{h}, u_{2}^{h}, g^{h}\right)=\frac{1}{2} \sum_{j=1}^{\ell+1}\left(u_{1 j}-u_{2 j}\right)^{2}+\frac{\delta}{2} \sum_{j=1}^{\ell+1} g_{j}^{2} .
$$

\subsection{Convergence and error estimation}

Let's consider the notations:

$$
\begin{array}{rlcl}
\mathcal{V}_{i} & = & H_{\Gamma_{i}}^{1}\left(\Omega_{i}\right)^{2} \cap H^{2}\left(\Omega_{i}\right)^{2} & i=1,2 \\
\mathbb{X} & = & \mathcal{V}_{1} \times \mathcal{V}_{2} \times L^{2}\left(\Gamma_{0}\right)^{2}, & \\
\mathbb{Y} & = & H^{2}\left(\Omega_{1}\right)^{2} \times H^{2}\left(\Omega_{2}\right)^{2} \times L^{2}\left(\Gamma_{0}\right)^{2}, \\
\mathbb{X}^{h} & = & \mathcal{V}_{1}^{h} \times \mathcal{V}_{2}^{h} \times \mathcal{W}^{h}, &
\end{array}
$$


The problem (1) has a unique solution $\left(u_{1}, u_{2}, g\right) \in \mathbb{X}[7]$.

Let $\pi_{h}: \mathbb{X} \rightarrow \mathbb{X}^{h}$ be the standard Lagrange interpolation operator that is depicted below

$$
\pi_{h}\left(u_{1}, u_{2}, g\right)=\left(u_{1}^{h}, u_{2}^{h}, g^{h}\right),
$$

through the theorem $I X .25$ [1, page 181] about the regularity of the Dirichlet problem, we have $u_{i} \in H^{2}\left(\Omega_{i}\right)(i=1,2)$, and there exists an integer $k$ and a constante $c$ independent of $h$ and $u$ such that [9]:

$$
\left\|u_{i}-u_{i}^{h}\right\|_{H^{1}\left(\Omega_{i}\right)^{2}} \leq c . h\left\|u_{i}\right\|_{H^{2}\left(\Omega_{i}\right)^{2}}, \quad \forall u_{i} \in H^{2}\left(\Omega_{i}\right)^{2}
$$

Indeed, the only argument in this theorem is the construction of an interpolation operator based on the characterization of functions in $\mathcal{V}_{i}^{h}$ by their values at the nodes, which is always possible for Lagrange's finite element:

$$
\begin{aligned}
\left\|\left(u_{1}, u_{2}, g\right)-\left(u_{1}^{h}, u_{2}^{h}, g^{h}\right)\right\|_{\mathbb{X}} & \leq c . h\left\|\left(u_{1}, u_{2}, g\right)\right\|_{\mathbb{Y}} \\
& \leq c . h\left(\sum_{i=1}^{2}\left\|u_{i}\right\|_{H^{2}\left(\Omega_{i}\right)^{2}}+\|g\|_{L^{2}\left(\Gamma_{0}\right)^{2}}\right),
\end{aligned}
$$

the coercivity of $a_{\rho_{i}}$ implies, $\exists c_{i}>0, \forall u_{i} \in \mathcal{V}_{i}(i=1,2)$ such that

$$
\begin{aligned}
\left\|u_{i}\right\|_{H^{2}\left(\Omega_{i}\right)^{2}}^{2} & \leq c_{i} \cdot a_{\rho_{i}}\left(u_{i}, u_{i}\right) \\
\Rightarrow\left\|u_{i}\right\|_{H^{2}\left(\Omega_{i}\right)^{2}}^{2} & \leq c_{i} \cdot\left(\int_{\Omega_{i}} f u_{i} d \Omega_{i}+(-1)^{i+1} \int_{\Gamma_{0}} g u_{i} d \Gamma_{0}\right) \\
\Rightarrow\left\|u_{i}\right\|_{H^{2}\left(\Omega_{i}\right)^{2}}^{2} & \leq c_{i} \cdot\left(\|f\|_{L^{2}\left(\Omega_{i}\right)^{2}}\left\|u_{i}\right\|_{H^{2}\left(\Omega_{i}\right)^{2}}+\|g\|_{L^{2}\left(\Gamma_{0}\right)^{2}}\left\|u_{i / \Gamma_{0}}\right\|_{L^{2}\left(\Gamma_{0}\right)^{2}}\right)
\end{aligned}
$$

where $u_{i / \Gamma_{0}}$ is the trace of $u_{i}$ on $\Gamma_{0}$. According to the trace theorem [1], we obtain

$$
\exists \lambda_{i}>0, i=1,2 \text { whereas }\left\|u_{i / \Gamma_{0}}\right\|_{L^{2}\left(\Gamma_{0}\right)^{2}} \leq \lambda_{i}\left\|u_{i}\right\|_{H^{2}\left(\Omega_{i}\right)^{2}}
$$

hence $\left\|u_{i}\right\|_{H^{2}\left(\Omega_{i}\right)^{2}} \leq c_{i}$. $\left(\|f\|_{L^{2}\left(\Omega_{i}\right)^{2}}+\lambda_{i}\|g\|_{L^{2}\left(\Gamma_{0}\right)^{2}}\right)$. The relation (18) is given by $\left(C=c \cdot \sup \left(c_{1}, c_{2}, \lambda_{1} c_{1}+\lambda_{2} c_{2}+1\right)\right)$ :

$$
\left\|\left(u_{1}, u_{2}, g\right)-\left(u_{1}^{h}, u_{2}^{h}, g^{h}\right)\right\|_{\mathbb{X}} \leq C . h\left(\|f\|_{L^{2}\left(\Omega_{1}\right)^{2}}+\|f\|_{L^{2}\left(\Omega_{2}\right)^{2}}+\|g\|_{L^{2}\left(\Gamma_{0}\right)^{2}}\right) .
$$

So, we find the desired convergence.

\section{Multicriteria approach to the problem (10)}

\subsection{Preliminary}

Definition 4.1. Let $M \subseteq \mathbb{R}^{m}$ be an arbitrary set, for any cone $K \subseteq M$, we define the binary relation:

$$
x \prec_{K} y \Leftrightarrow y-x \in K,
$$


Definition 4.2. A vector $\bar{y} \in M$ is nondominated in $M$ w.r.t $\prec_{K}$, if $\nexists y$ satisfies $y \prec_{K} \bar{y}$. The set of all nondominated points or efficient points denoted

$$
E_{f f}(M, K)=\left\{y \in M \mid y \text { nondominated in } M \text { w.r.t } \prec_{K}\right\} .
$$

The set of feasible points of the lower-level problem

$$
\Lambda=\left\{g^{h} \in \mathcal{W}^{h} \mid A_{1}^{h} \cdot u_{1}^{h}=b_{1}^{h}, A_{2}^{h} \cdot u_{2}^{h}=b_{2}^{h}\right\},
$$

Now, $x=\left(u^{h}, g^{h}\right) \in E=\mathbb{R}^{4 N} \times \mathcal{W}^{h}$, solves (10)

$\Longleftrightarrow \quad x$ feasible and $\quad \nexists y=\left(v^{h}, g_{1}^{h}\right) \in E: \quad y$ feasible and $\ell(y)<\ell(x)$

$\Longleftrightarrow \quad \nexists \bar{g}^{h} \in \mathcal{W}^{h}: \quad \mathcal{J}_{\delta}\left(u^{h}, \bar{g}^{h}\right)<\mathcal{J}_{\delta}(x)$

and $\nexists y=\left(v^{h}, g_{1}^{h}\right) \in E: \quad g_{1}^{h} \in \arg \min _{z \in \Lambda} \mathcal{J}_{\delta}\left(v^{h}, z\right)$ and $\ell(y)<\ell(x)$

The function $\mathcal{J}_{\delta}\left(u^{h},.\right)$ is convex then $x=\left(u^{h}, g^{h}\right) \in E=\mathbb{R}^{4 N} \times \mathcal{W}^{h}$ doesn't solve (10) thus is equivalent to $\exists \bar{g}^{h} \in \mathcal{W}^{h}$ such that $\mathcal{J}_{\delta}\left(u^{h}, \bar{g}^{h}\right)<\mathcal{J}_{\delta}(x)$, or

$$
\exists y=\left(v^{h}, g_{1}^{h}\right) \in E: \quad\left\|P\left(\nabla_{2} \mathcal{J}_{\delta}(x)\right)\right\|=0 \text { and } \ell(y)<\ell(x)
$$

where $P($.$) is the projection operator [2] onto \Lambda$ and $\nabla_{2} \mathcal{J}_{\delta}(x)$ is the first derivative of $\mathcal{J}_{\delta}\left(u^{h}, g^{h}\right)$ with respect to the second argument $g^{h}$.

For $x=\left(u^{h}, g_{1}^{h}\right), y=\left(v^{h}, g_{2}^{h}\right) \in E=\mathbb{R}^{4 N} \times \mathcal{W}^{h}$, we define the relation

$$
\begin{array}{ccc}
x \prec y & \Longleftrightarrow \quad\left[u^{h}=v^{h} \text { and } \mathcal{J}_{\delta}(x)<\mathcal{J}_{\delta}(y)\right] \\
& \Longleftrightarrow \quad\left[\left\|P\left(\nabla_{2} \mathcal{J}_{\delta}(x)\right)\right\|=0 \text { and } \ell(x)<\ell(y)\right] .
\end{array}
$$

\subsection{Main results}

From the definition of $\prec$ above, we conclude the fundamental theorem of this paper.

Theorem 4.3. $x^{*}=\left(u^{* h}, g^{* h}\right) \in$ E solves the two-levels programming problem (10) if and only if $x^{*}$ is nondominated with respect to $\prec$.

Proof. $x^{*}=\left(u^{* h}, g^{* h}\right)$ solves the two-levels programming problem (10),

$$
\begin{array}{ccc}
\Longleftrightarrow \quad \nexists \bar{g}^{h} \in \mathcal{W}^{h}: \quad \mathcal{J}_{\delta}\left(u^{* h}, \bar{g}^{h}\right)<\mathcal{J}_{\delta}\left(x^{*}\right) \\
\text { and } \nexists y=\left(v^{h}, g_{1}^{h}\right) \in E: \quad g_{1}^{h} \in \arg \min _{z \in \Lambda} \mathcal{J}_{\delta}\left(v^{h}, z\right) \quad \text { and } \ell(y)<\ell\left(x^{*}\right)
\end{array}
$$

it follows that $\nexists \bar{x}=\left(u^{* h}, \bar{g}^{h}\right) \in E: \mathcal{J}_{\delta}(\bar{x})<\mathcal{J}_{\delta}\left(x^{*}\right)$, thus, $x^{*}$ is nondominated with respect to $\prec$.

Conversely, assume that $x^{*}$ doesn't solve the two-levels programming problem (10), then

$$
\begin{gathered}
\exists \bar{g}^{h} \in \mathcal{W}^{h}: \quad \mathcal{J}_{\delta}\left(u^{* h}, \bar{g}^{h}\right)<\mathcal{J}_{\delta}\left(x^{*}\right) \\
\text { or } \quad \exists y=\left(v^{h}, g_{1}^{h}\right) \in E: \quad g_{1}^{h} \in \arg \min _{z \in \Lambda} \mathcal{J}_{\delta}\left(v^{h}, z\right) \quad \text { and } \quad \ell(y)<\ell\left(x^{*}\right)
\end{gathered}
$$

hence, we have $\exists \bar{x}=\left(u^{* h}, \bar{g}^{h}\right) \in E: \mathcal{J}_{\delta}(\bar{x})<\mathcal{J}_{\delta}\left(x^{*}\right)$ which contradicts the fact that $x^{*}$ is nondominated with respect to $\prec$. 


\subsection{Problem statement}

Definition 4.4. Let $M \subseteq \mathbb{R}^{m}$ be a set, a function $v: M \rightarrow \mathbb{R}$ is called $K-$ monotonically increasing in $M$ if,

$$
a \prec_{K} b \Rightarrow v(a) \leq v(b) \text { for all } a, b \in M \text {. }
$$

$v$ is called strictly $K$-monotonically increasing in $M$ if,

$$
a \prec_{K} b \text { and } a \neq b \Rightarrow v(a)<v(b) \text { for all } a, b \in M \text {. }
$$

Theorem 4.5. Let $K \subset \mathbb{R}^{m}$ be a cone with $\{0\} \neq K \neq \mathbb{R}^{n}$. Let $v$ be a $K$ - monotone increasing function and let $a \in M$ be a minimum of $v$ over $M$. If a is unique or if $v$ is strictly $K$ - monotone increasing in $M$, then $a$ is nondominated in $M$ with respect to $\prec_{K}$.

Proof. Suppose for contradiction that $a \notin E_{f f}(M, K)$, thus $\exists b \in M$ such that $b \neq a$ and $b \prec_{K} a$.

Since $v$ is $K-$ monotone increasing, then $v(b) \leq v(a)$, it follows that

$b \in \arg \min _{y \in M} v(y)$

if $a$ is unique, then contradiction.

if $v$ is strictly $K-$ monotone increasing in $M$, then $v(b)<v(a)$, so $a$ is not a minimum of $v$ over $M$, and we have a contradiction.

Definition 4.6. Let $K \subset \mathbb{R}^{m}$ be a cone and $M$ a set. The dual cone of $K$ is defined by

$$
K^{*}=\left\{y \in \mathbb{R}^{m} \mid \forall k \in K:\langle y, k\rangle \geq 0\right\}
$$

the quasi-interior of $K^{*}$ is defined by

$$
K^{+}=\left\{y \in \mathbb{R}^{m}, y \neq 0 \mid \forall k \in K:\langle y, k\rangle>0\right\}
$$

with $\langle.,$.$\rangle the (standard) inner product in \mathbb{R}^{m}$.

For a matrix $Q \in \mathbb{R}^{m \times m}$, we note $Q M=\{Q x \mid x \in M\}$.

The set $M$ that we will use in what follows will be the image set of the function $F$ defined by:

$$
\begin{array}{cccl}
F: & E & \rightarrow & M \\
x= & \left(u^{h}, g^{h}\right) & \mapsto & \left(u^{h}, \ell(x), \mathcal{J}_{\delta}(x),\left\|P\left(\nabla_{2} \mathcal{J}_{\delta}(x)\right)\right\|\right),
\end{array}
$$

then $M=\mathbb{R}^{4 N} \times \mathbb{R} \times \mathbb{R}_{+} \times \mathbb{R}_{+}$. We consider the multicriteria optimization problem (MOP)

$$
\min _{x \in E} F(x)=\left(u^{h}, \ell(x), \mathcal{J}_{\delta}(x),\left\|P\left(\nabla_{2} \mathcal{J}_{\delta}(x)\right)\right\|\right),
$$


Consider the cone

$$
K=\left\{\left(u^{h}, r, s, t\right) \in M /\left(u^{h}=0_{\mathbb{R}^{4 N}} \text { and } s>0\right) \text { or }(r>0 \text { and } t \geq 0)\right\} .
$$

The cone $K$ is not convex, let us define two convex cones $K_{1}$ and $K_{2}$ :

$$
\begin{gathered}
K_{1}=\left\{\left(u^{h}, r, s, t\right) \in M / u^{h}=0_{\mathbb{R}^{4 N}} \text { and } s>0\right\}, \\
K_{2}=\left\{\left(u^{h}, r, s, t\right) \in M / r>0 \text { and } t \geq 0\right\},
\end{gathered}
$$

thus

$$
\begin{array}{ccc}
K_{1} & = & \left\{0_{\mathbb{R}^{4 N}}\right\} \times \mathbb{R} \times \mathbb{R}_{+}^{*} \times \mathbb{R}_{+}, \\
K_{2} & = & \mathbb{R}^{4 N} \times \mathbb{R}_{+}^{*} \times \mathbb{R}_{+} \times \mathbb{R}_{+} \\
K & = & K_{1} \cup K_{2},
\end{array}
$$

then, the dual cones are:

$$
\begin{aligned}
& K_{1}^{*}=\mathbb{R}^{4 N} \times\{0\} \times \mathbb{R}_{+} \times \mathbb{R}_{+}, \\
& K_{2}^{*}=\left\{0_{\mathbb{R}^{4 N}}\right\} \times \mathbb{R}_{+} \times \mathbb{R}_{+} \times \mathbb{R}_{+} .
\end{aligned}
$$

Theorem 4.7. Let $K \subset \mathbb{R}^{m}$ be a convex cone and $M \subset \mathbb{R}^{m}$ be a convex set and $L$ be the subspace parallel to af $f(M)$. Let $Q \subseteq \mathbb{R}^{m \times m}$ be a positive definite matrix, we define the function $v(x)=\langle Q x, x\rangle \forall x \in M$. If $Q M \subset K^{*}+L^{\perp}$, then $v$ is monotonically increasing with respect to $\prec_{K}$ on $M$.

Proof. $\forall x \in M, Q M \subset K^{*}+L^{\perp}=(K \cap L)^{*} \Rightarrow\langle Q x, y\rangle \geq 0, \forall y \in K \cap L$, furthermore, as $\nabla v(x)=2 Q x \quad \forall x$, then $v$ is monotonically increasing with respect to $\prec_{K}$ on $M$, indeed if $x \prec_{K} z$

$\Longrightarrow-x+z \in K \cap(-x+M)$ then, $-x+z \in K \cap L$

$\Longrightarrow\langle Q x,-x+z\rangle \geq 0$ since $Q M \subset(K \cap L)^{*}$

$\Longrightarrow\langle Q x, x\rangle \leq\langle Q x, z\rangle$ or also, $v(x) \leq\langle Q x, z\rangle$

furthermore, $\langle Q(z-x), z\rangle \geq 0$, for all $z \in K \cap L$, because $K \subset M$ (definition of $K(32)$ ), thus $\langle Q x, z\rangle \leq v(z)$, it turns out that $v$ is monotonically increasing with respect to $\prec_{K}$ on $M$.

\subsection{Quadratic scalarizations}

For the sake of simplicity, we consider the diagonal matrices $Q=\operatorname{diag}\left(\alpha_{1}, \alpha_{2}, \alpha_{3}, \alpha_{4}, \alpha_{5}\right)$ then we have the following results.

Proposition 4.8. Using the above notations, let $Q \in \mathbb{R}^{m \times m}$ be a positive definite matrix and define the function $v$ by $v(y)=\langle Q y, y\rangle$, if $y \in K^{+}$then $v$ is strictly $K$-monotone increasing. 
Proof. Let $v$ be the function defined by $v(y)=\langle Q y, y\rangle, \forall y \in M$, $\forall y_{1}, y_{2} \in M, y_{i} \in K^{+} \Longrightarrow Q y_{i} \in K^{+}$suppose that: $y_{1} \prec_{K} y_{2}$

$$
\begin{array}{cc}
\Longrightarrow & y_{2}-y_{1} \in K \\
\Longrightarrow & \left\langle Q y_{2}, y_{2}-y_{1}\right\rangle>0 \\
\Longrightarrow & \left\langle Q y_{2}, y_{1}\right\rangle<\left\langle Q y_{2}, y_{2}\right\rangle \\
\Longrightarrow & \left\langle Q y_{2}, y_{1}\right\rangle<v\left(y_{2}\right),
\end{array}
$$

furthermore $\left\langle Q\left(y_{2}-y_{1}\right), y_{1}\right\rangle>0$

$$
\begin{array}{cc}
\Longrightarrow & \left\langle Q y_{1}, y_{1}\right\rangle<\left\langle Q y_{2}, y_{1}\right\rangle \\
\Longrightarrow & v\left(y_{1}\right)<\left\langle Q y_{2}, y_{1}\right\rangle, \\
\Longrightarrow & v\left(y_{1}\right)<v\left(y_{2}\right),
\end{array}
$$

thus, $v$ is strictly $K$-monotone increasing.

Theorem 4.9. Let $K$ be a convex cone and $M$ be a convex set with nonempty interior. For a positive definite matrix $Q \subseteq \mathbb{R}^{m \times m}$ with $Q M \subset K^{*}$,

$$
\text { if } y^{*} \in \arg \min _{y \in M}\langle Q y, y\rangle \text { then } y^{*} \in E_{f f}(M, K) \text {. }
$$

Proof. Let $y^{*} \in \arg \min _{y \in M} v(y)$ with $v(y)=\langle Q y, y\rangle$, then $\forall y \in M \quad v\left(y^{*}\right) \leq$ $v(y)$, if $y^{*} \notin E_{f f}(M, K)$, then $\exists \bar{y} \in M, \bar{y} \neq y^{*}$ and $\bar{y} \prec_{K} y^{*}$, since $v$ is $K$-monotone increasing in $M$ according to the Theorem 4.7, it follows that $v\left(y^{*}\right) \geq v(\bar{y})$, this implies that $v\left(y^{*}\right)=v(\bar{y})$ and $\bar{y} \neq y^{*}$ contradiction with uniqness of the minimum of $v(y)$ since $v$ is strictly convex.

Theorem 4.10. If $y^{*}=F\left(u^{* h}, g^{* h}\right) \in M$ is nondominated with respect to $\prec_{K}$, for some $\left(u^{* h}, g^{* h}\right) \in \mathbb{R}^{4 N} \times \mathcal{W}^{h}$, then $x^{*}=\left(u^{* h}, g^{* h}\right)$ is nondominated w.r.t $\prec$.

Proof. $y^{*}=F\left(x^{*}\right) \in M$ is nondominated with respect to $\prec_{K}$,

$\Longrightarrow \nexists \bar{y}=F(\bar{x}) \in M: \bar{y} \prec_{K} y^{*}$

$\Longrightarrow \nexists \bar{y} \in M: y^{*}-\bar{y} \in K$

$\Longrightarrow \nexists \bar{x} \in E:$

$$
\begin{gathered}
{\left[\bar{u}^{h}=u^{* h} \text { and } \mathcal{J}_{\delta}(\bar{x})<\mathcal{J}_{\delta}\left(x^{*}\right)\right]} \\
\text { or }\left[\left\|P\left(\nabla_{2} \mathcal{J}_{\delta}(\bar{x})\right)\right\| \leq\left\|P\left(\nabla_{2} \mathcal{J}_{\delta}\left(x^{*}\right)\right)\right\| \quad \text { and } \quad \ell(\bar{x})<\ell\left(x^{*}\right)\right],
\end{gathered}
$$

we have necessarily $P\left(\nabla_{2} \mathcal{J}_{\delta}\left(x^{*}\right)\right)=0$. For contradiction, assume that $P\left(\nabla_{2} \mathcal{J}_{\delta}\left(x^{*}\right)\right) \neq 0$, then $x^{*}$ is not a stationnary point [2], will implies that $x^{*} \notin \arg \min _{x \in \Lambda} \mathcal{J}_{\delta}(x)$, thus $\exists \bar{x} \in E: \bar{x} \in \Lambda$ and $\mathcal{J}_{\delta}(\bar{x})<\mathcal{J}_{\delta}\left(x^{*}\right)$, contradicting with (36). Hence, $\nexists \bar{x} \in E$ :

$$
\begin{gathered}
{\left[\bar{u}^{h}=u^{* h} \text { and } \mathcal{J}_{\delta}(\bar{x})<\mathcal{J}_{\delta}\left(x^{*}\right)\right]} \\
\text { or }\left[\left\|P\left(\nabla_{2} \mathcal{J}_{\delta}(\bar{x})\right)\right\|=0 \text { and } \ell(\bar{x})<\ell\left(x^{*}\right)\right],
\end{gathered}
$$

consequently, $\nexists \bar{x} \in E$ such that $\bar{x} \prec x^{*}$, which implies that $x^{*}$ is nondominated w.r.t $\prec$. 
For $x=\left(u^{h}, g^{h}\right)$, we have $Q M \subseteq K_{1}^{*}$ implies

$$
\begin{array}{ccc}
\operatorname{voF}\left(u^{h}, g^{h}\right) & = & v\left(u^{h}, \ell(x), \mathcal{J}_{\delta}(x),\left\|P\left(\nabla_{2} \mathcal{J}_{\delta}(x)\right)\right\|\right), \text { for } u^{h}=\left(u_{1}^{h}, u_{2}^{h}\right) \\
v(y) & = & \alpha_{1}\left(u_{1}^{h}\right)^{2}+\alpha_{2}\left(u_{2}^{h}\right)^{2}+\alpha_{4} \mathcal{J}_{\delta}^{2}\left(u^{h}, g^{h}\right)+\alpha_{5}\|\bar{g}\|^{2}
\end{array}
$$

such that $P\left(\nabla_{2} \mathcal{J}_{\delta}\left(u^{h}, g^{h}\right)\right)=\bar{g}=\arg \min _{\hat{g} \in \Lambda}\left\|\delta g^{h}-\hat{g}\right\|$.

Similarly, we have: $Q M \subseteq K_{2}^{*}$ implies

$$
\begin{array}{ccc}
\operatorname{voF}\left(u^{h}, g^{h}\right) & = & v\left(u^{h}, \ell(x), \mathcal{J}_{\delta}(x),\left\|P\left(\nabla_{2} \mathcal{J}_{\delta}(x)\right)\right\|\right) \\
v(y) & = & \alpha_{3} \ell^{2}\left(u^{h}, g^{h}\right)+\alpha_{4} \mathcal{J}_{\delta}^{2}\left(u^{h}, g^{h}\right)+\alpha_{5}\left\|P\left(\nabla_{2} \mathcal{J}_{\delta}(x)\right)\right\|^{2} \\
& = & \alpha_{3} \ell^{2}\left(u^{h}, g^{h}\right)+\alpha_{4} \mathcal{J}_{\delta}^{2}\left(u^{h}, g^{h}\right)+\alpha_{5}\|\bar{g}\|^{2}
\end{array}
$$

Consequently, we solve two single optimization problems

$$
\min _{y \in M} v(y)
$$

The objective function $v$ is bounded below for all feasible parameter values $\alpha_{i}>0, i=1,5$ ( $Q$ positive definite), and since $v$ is strictly convex and monotonically increasing w.r.t $\prec_{K_{i}}$, then the minimization problem (40) has a unique solution $y^{*}=F\left(x^{*}\right)$, and according to the Theorem 4.7 , this minimum is a nondominated point w.r.t $\prec_{K_{i}}$. Each choice of parameters $\alpha_{i}$ gives a unique solution $y^{*}$, consequently, to generate $E_{f f}\left(M, K_{i}\right)$ we would have to choose a finite set of parameters [4].

As $K$ is not convex, a natural question is how to characterize the efficient set w.r.t. $\prec_{K}, E_{f f}(M, K)$, and then how to compute the Pareto optimal points which are the optimal solutions of the BPP. The following theorem by Pieume et al. [8] gives an answer to this question.

\section{Proposition 4.11.}

$$
E_{f f}(M, K)=E_{f f}\left(M, K_{1}\right) \cap E_{f f}\left(M, K_{2}\right)
$$

Proof. Let $x \notin E_{f f}(M, K)$

$$
\begin{aligned}
& \Leftrightarrow \exists y \in M, \quad y \prec_{K} x \\
& \Leftrightarrow \exists y \in M, \quad x-y \in K, \\
& \left.\Leftrightarrow \exists y \in M, \quad x-y \in K_{1} \text { or } x-y \in K_{2} \quad \text { (because } K=K_{1} \cup K_{2}\right), \\
& \Leftrightarrow \exists y \in M, \quad y \prec_{K_{1}} x \text { or } y \prec_{K_{2}} x, \\
& \Leftrightarrow \quad\left(x \notin E_{f f}\left(M, K_{1}\right)\right) \text { or }\left(x \notin E_{f f}\left(M, K_{2}\right)\right), \\
& \Leftrightarrow x \notin E_{f f}\left(M, K_{1}\right) \cap E_{f f}\left(M, K_{2}\right) .
\end{aligned}
$$


That is, for any point $y^{*}=F\left(x^{*}\right)$ belonging to the intersection of the two efficient sets $E_{f f}\left(M, K_{1}\right) \cap E_{f f}\left(M, K_{2}\right)$, the corresponding Pareto optimal solution $x^{*}=\left(u^{* h}, g^{* h}\right)$ is an optimal solution to the approximated $\operatorname{BPP}\left(P_{h}\right)$. This result is applicable if and only if $E_{f f}(M, K) \neq \emptyset$, otherwise, the relationship between multicriteria and bi-level programming is not applicable to solve BPP.

\section{Conclusions and Future work}

We can use multiobjective optimization techniques successfully to solve bilevel programs. Among the most popular methods, the scalarization techniques plays a crucial role for solving multicriteria problems.

In this work, we have established mathematically a new solution of decomposed topology optimization problem using finite element approximation. It is also presented a relationship between DTOP written as bilevel programming problem, and the multicriteria optimization, generally difficult to justify. With this mathematical result, one can to take advantage of multicriteria techniques in order to solving the approximated DTOP.

The aim of the future research is to propose an algorithm to make a numerical solution for academic purpose, and then improve the proposed method for industrial cases.

\section{References}

[1] H. Brézis, Analyse fonctionnelle, Théorie et Applications, Masson, Paris, 1983.

[2] P. H. Calamai and J. J. More, Projected Gradient Methods for Linearly Constrained Problems, Mathematical Programming, 39 (1987), 93-116.

[3] A. El Hami and B. Radi, Incertitudes, optimisation et fiabilite des structures, Hermès-Lavoisier, Paris, 2013.

[4] J. Fliege and L. N. Vicente, Multicriteria Approach to Bilevel Optimization, Journal of optimization theory and applications, 131 (2006), 209225 .

[5] J. Fulop, On the equivalence between a linear bilevel programming problem and linear optimization over the efficient set, Technical report WP931, Laboratory of Operations Research and Decision Systems, Computer and Automation Institute, Hungarian Academy of Sciences, 1993. 
[6] A. Makrizi, B. Radi and A. El Hami, Solution of the topology optimization problem based subdomain method, Applied Mathematical Sciences, 2 (2008), 2029-2045.

[7] A. Makrizi and B. Radi, Bilevel approach of a decomposed topology optimization problem, Mathematical Modelling of Natural Phenomena, 5 (2010), 128-131.

[8] C. O. Pieume, L. P. Fotso and P. Siarry, Solving bilevel programming problems with multicriteria optimization techniques, Op. Research, 46 (2009), 169-183.

[9] P.A. Raviart and J.M. Thomas, Introduction à l'analyse numérique des équations aux dérivées partielles, Masson, Paris, 1983.

[10] H.V. Stackelberg, The theory of market economy, Oxford University Press,Oxford, 1952.

Received: January 3, 2014 